\title{
A Nontrivial Renormalization Group Fixed Point for the Dyson-Baker Hierarchical Model
}

\author{
Hans Koch ${ }^{1}$, Peter Wittwer ${ }^{2}$ \\ 1 Supported in Part by the National Science Foundation under Grant No. DMS-9103590. Department \\ of Mathematics, University of Texas at Austin, Austin, TX 78712, USA \\ 2 Supported in Part by the Swiss National Science Foundation. Département de Physique Théorique, \\ Université de Genève, Genève, $\mathrm{CH}$ 1211, Switzerland
}

Received: 15 September 1993

\begin{abstract}
We prove the existence of a nontrivial Renormalization Group ( $R G$ ) fixed point for the Dyson-Baker hierarchical model in $d=3$ dimensions. The single spin distribution of the fixed point is shown to be entire analytic, and bounded by $\exp \left(-\right.$ const $\left.\times t^{6}\right)$ for large real values of the spin $t$. Our proof is based on estimates for the zeros of a RG fixed point for Gallavotti's hierarchical model. We also present some general results for the heat flow on a space of entire functions, including an order preserving property for zeros, which is used in the RG analysis.
\end{abstract}

\section{Introduction and Main Results}

One of the basic assumptions in the modern theory of critical phenomena is the existence of nontrivial renormalization group (RG) fixed points, associated with certain universality classes of interactions. Within the framework of statistical mechanics, this assumption is actually a conjecture, and it should be possible to either prove it or disprove it. However, even for the simplest classes of "realistic" interactions, such as the one represented by the three-dimensional Ising model, the rigorous construction of a nontrivial $R G$ fixed point seems beyond the reach of presently known methods. In addition, there is a lack of good numerical results in this area, which indicates that even at a quite fundamental level there are still gaps in our understanding of RG transformations.

The traditional approach in such situations is to try to first understand some simpler, and thus necessarily less realistic, class of interactions. In this case, the model with the longest history is Dyson's hierarchical model [1-4]. For the Dyson-type hierarchical analogue of the nearest-neighbor (continuous spin) Ising model in $d=3$ dimensions, which was first considered by Baker [3], the full RG transformation $\mathscr{T}$ reduces to the third power of the following nonlinear operator $\mathscr{R}$ :

$$
(\mathscr{R}(h))(t)=K \int_{-\infty}^{\infty} d x e^{-2 \sigma x^{2}} h(\alpha t+x) h(\alpha t-x), \quad t \in \mathbb{R}
$$


Here, $h$ is the density of the single spin distribution, $K$ and $\sigma$ are arbitrary but fixed positive real numbers, and

$$
\alpha=2^{-\frac{d-2+\eta}{2 d}}=2^{-\frac{1}{6}}, \quad(d=3, \eta=0) .
$$

More details about the connection between the transformations $\mathscr{T}$ and $\mathscr{B}$ will be given in Section 5. Our main result is the following.

Theorem 1.1. $\mathscr{R}$ has a fixed point which is the restriction to $\mathbb{R}$ of an even entire analytic function $h_{I R}$. Given any $\gamma>6$, the function $h_{I R}$ satisfies the bounds

$$
\begin{aligned}
0<h_{I R}(t) & <c_{1} e^{-c_{2} t^{6}}, & \forall t & \in \mathbb{R}, \\
\left|h_{I R}(t)\right| & <c_{3} e^{c_{4}|t|^{\gamma}}, & \forall t & \in \mathbb{C} .
\end{aligned}
$$

for some positive constants $c_{1}, \ldots, c_{4}$.

In what follows, we choose the values $K=2 \alpha$ and $\sigma=\left(2 \alpha^{2}-1\right) /\left(4 \alpha^{2}-1\right)$ for the normalization constants that appear in the definition of $\mathscr{R}$. This can be done without loss of generality, since any two transformations of the type (1) are conjugate via a scaling of the form $h \mapsto a h(b$.).

Let now $h_{I R}$ be the fixed point of $\mathscr{B}$ described in Theorem 1.1. Then we can define an entire analytic function $f_{I R}$ by the equation

$$
h_{I R}(t)=e^{\sigma t^{2}} \frac{\sigma}{\pi} \int_{-\infty}^{\infty} d s e^{-2 i \sigma s t} f_{I R}(i s), \quad t \in \mathbb{R} .
$$

A straightforward calculation shows that $f_{I R}$ satisfies the fixed point equation for the following transformation $\mathscr{N}$ :

$$
(\mathscr{N}(f))(t)=\frac{1}{\sqrt{\left(1-\beta^{2}\right) \pi}} \int_{-\infty}^{\infty} d s e^{-\frac{1}{1-\beta^{2}} s^{2}} f(\beta t+s)^{2}, \quad t \in \mathbb{C},
$$

with $\beta=\frac{1}{2 \alpha}$. $=2^{-5 / 6}$. Conversely, if $f_{I R}$ is some given fixed point for $\mathscr{N}$, one can try to use Eq. (1.5) in order to obtain a fixed point $h_{I R}$ for $\mathscr{B}$. This is exactly the strategy which we have adopted here. The main problem is to estimate the function $f_{I R}$ along the imaginary axis. The following lemma, together with Eq. (1.5), implies Theorem 1.1.

Lemma 1.2. $\mathscr{N}$ has a fixed point $f_{I R}$ with the following properties. $f_{I R}$ is entire analytic, $f_{I R}(i$.$) is a function of positive type (i.e., the Fourier transform of a finite$ positive measure), and for every positive $q<6 / 5$ there are constants $b_{1}, \ldots, b_{4}>0$ such that

$$
\begin{aligned}
\left|f_{I R}(t)\right|<b_{1} e^{b_{2}|t|^{6 / 5}}, & \forall t \in \mathbb{C}, \\
\left|f_{I R}(i t)\right|<b_{3} e^{-b_{4}|t|^{q}}, & \forall t \in \mathbb{R} .
\end{aligned}
$$

We note that the same result, but without the bound (1.8), was already obtained in [7]. To prove only that part of Lemma 1.2 , the analysis can be restricted, modulo a finite dimensional problem, to a neighborhood of the high temperature fixed point $f_{H T} \equiv 1$. The result is sufficient to show that there exists a nontrivial "weak" solution of the fixed point equation for $\mathscr{R}$. But in order to establish the regularity of this solution, as 
implied by the bound (1.8), it becomes necessary to analyze the transformation $\mathscr{N}$ on a more global scale. Roughly speaking, the infrared fixed point $h_{I R}$ of $\mathscr{R}$ inherits its asymptotic behavior (fast decay) from the high temperature fixed point $h_{H T}(t)=\delta(t)$, and its local regularity from the ultraviolet fixed point $h_{U V}=$ const. This is indicated e.g. by the fact that the fixed point $f_{I R}$ is "close" to $f_{H T}$ only with respect to its relatively slow growth; its behavior (1.8) along the imaginary axis is qualitatively much closer to that of the ultraviolet fixed point $f_{U V}(t)=\exp \left(\sigma t^{2}+\right.$ const). This may be regarded as a consequence of the generally believed fact (which we think could be proved with the methods developed here) that the ultraviolet fixed point lies on the boundary of the stable manifold of the infrared fixed point.

As was already shown in [7], the transformation. $\mathscr{N}$ has the following interesting property $P$ : If all zeros of a polynomial $f$ lie on the imaginary axis, then the same is true for $\mathscr{N}^{\circ}(f)$. In addition, we have the bound (1.7) on the growth of $f_{I R}$. Thus, since it seems likely that there exists at least one polynomial (e.g. close to the ultraviolet fixed point) which has only imaginary zeros, and which lies on the stable manifold of the fixed point $f_{I R}$, it is natural to conjecture that the infrared fixed point of $\mathscr{V}^{\text {. }}$ can be written as a canonical product

$$
f_{I R}(t)=f_{I R}(0) \prod_{k=0}^{\infty}\left[1+\left(\frac{t}{\nu_{k}}\right)^{2}\right], \quad t \in \mathbb{C},
$$

with purely imaginary zeros $\pm i \nu_{0}, \pm i \nu_{1}, \ldots$ This conjecture is indeed correct, but it appears very hard to prove. In fact, our first attempt contained an error [7, Lemma 4.2]. A new proof, which follows the argument given above (not for $\mathscr{N}$, but for a contraction with the same property $P$ and the same fixed point $\left.f_{I R}\right)$ can be found in [8].

Theorem 1.3. $\mathcal{H}$ has a fixed point $f_{I R}$ of the form (1.9), with $0<\nu_{0}<\nu_{1}<\ldots$ and $f_{I R}(0)>0$. Furthermore, given any $p<\frac{1}{6}$, there are positive constants $a_{1}$ and $a_{2}$ such that for $k=1,2, \ldots$

$$
\nu_{k}>a_{1} k^{5 / 6}, \quad \nu_{k}-\nu_{k-1}<a_{2} k^{-p} .
$$

This theorem implies Lemma 1.2., even though for a general product of the form (1.9), the bounds (1.10) are not sufficient to guarantee decay along the imaginary axis. In order to get (1.8), we also need to use that $f_{I R}$ is a fixed point of $\mathscr{N}$.

Our proof of Theorem 1.3 uses input (see Theorem 3.2) from a computer-assisted analysis, which is described in detail in [8]. In particular, it requires upper bounds on the first 80 gaps $\gamma_{k}=\nu_{k}-\nu_{k-1}$ for the fixed point $f_{I R}$ of . $\mathscr{N}$. Given these bounds, we use an order preserving property (for zeros) of the heat flow, and estimates on the evolution of polynomial approximations to the hyperbolic functions, in order to inductively estimate the gaps $\gamma_{2 k}$ and $\gamma_{2 k+1}$ in terms of the gaps near $\nu_{k}$. This method is suggested by a connection between the heat flow and the evolution of a system of interacting charges on a line.

The results stated so far will be proved in Sect. 3. We shall now describe some general results on the one-dimensional heat flow, which we use in our RG analysis, but which may be of independent interest. The proofs and further details will be given in Sect. 4.

Denote by $\mathscr{E}$ the set of all entire analytic functions $f$ that satisfy

$$
\varlimsup_{|z| \rightarrow \infty}|z|^{-2} \ln |f(z)|=0
$$


and whose zeros all lie on a straight line $\mu(f)+i \mathbb{R}$ parallel to the imaginary axis. For every $f \in \mathscr{E}$ and for every $\lambda>0$, define a function $H_{\lambda} f$ by the equation

$$
\left(H_{\lambda} f\right)(z)=\frac{1}{\sqrt{4 \pi \lambda}} \int_{-\infty}^{\infty} d s e^{-\frac{1}{4 \lambda} s^{2}} f(z-s), \quad z \in \mathbb{C}
$$

and let $H_{0} f=f$.

Lemma 1.4. Let $f \in \mathscr{E}$. Then for all $\lambda>0$, the function $H_{\lambda} f$ lies in $\mathscr{E}$ and has no multiple zeros. The set of solutions $(\lambda, z)$ of the equation $\left(H_{\lambda} f\right)(z)=0$ is a union of curves $\lambda \mapsto z(\lambda)$ which are continuous on $[0, \infty)$ and real analytic on $(0, \infty)$.

In what follows, we will assume that the zeros of a function $f \in \mathscr{E}$ have been arranged into an indexed set $\left\{\mu(f)+i \nu_{k}(f)\right\}_{k \in I}$, where multiple zeros are repeated according to their multiplicities. We also assume that $I$ is a set of consecutive integers, and that the zeros are labeled in such a way that the gaps

$$
\gamma_{k}(f)= \begin{cases}\nu_{k}(f)-\nu_{k-1}(f), & \text { if }\{k-1, k\} \subset I \\ +\infty, & \text { otherwise }\end{cases}
$$

are nonnegative, for all integers $k$. When indexed this way, the zeros of $f$ determine a double-sided sequence $\gamma(f)$ of numbers in $[0, \infty]$. On the set of such sequences, one has the following canonical partial order relation.

Definition 1.5. $\gamma \prec \gamma^{\prime} \Longleftrightarrow \gamma_{k} \leq \gamma_{k}^{\prime}, \forall k \in \mathbb{Z}$.

We note that by Lemma 1.4 , if $f$ is a function in $\mathscr{E}$ with zeros indexed by $I$, then there exists a canonical (unique) way of indexing the zeros of the entire family $\left\{H_{\lambda} f\right\}_{\lambda \geq 0}$ by the same set $I$, in such a way that the curves $\lambda \mapsto \nu_{k}\left(H_{\lambda} f\right)$ are continuous for all $k \in I$.

Theorem 1.6. Let $f$ and $g$ be functions in $\mathscr{E}$ with indexed zeros. If the functions $H_{\lambda} f$ and $H_{\lambda} g$ are indexed canonically, then $\gamma(f) \prec \gamma(g)$ implies $\gamma\left(H_{\lambda} f\right) \prec \gamma\left(H_{\lambda} g\right)$, for all $\lambda>0$.

\section{Remarks.}

- In [8] it is shown that the fixed point $f_{I R}$ described here is unique in a small open subset $\odot$ of some Banach space. Previously, in [7, Sect. 3], we proved the existence of a nontrivial fixed point for $\mathscr{N}$ in some other set $G \oplus H$. We have no doubt that $(2)$ is contained in $G \oplus H$, but we did not try to prove this, since we have no uniqueness result on $G \oplus H$. On the other hand, the fixed point properties derived in [7, Sect. 4] hold for any non-constant fixed point of $\mathscr{N}$ in a function space that is much larger than (and contains) the spaces considered here. Thus, all these results apply to $f_{I R}$. This includes the following stronger version of the estimate (1.7). If we define $\ell_{n}\left(t^{2}\right)=\left(\beta^{-n} t\right)^{-6 / 5} \ln f_{I R}\left(\beta^{-n} t\right)$, then the limit $\ell(x)=\lim _{n \rightarrow \infty} \ell_{n}(x)$ exists and is nonzero for all $x \in \mathbb{R}_{+}$, and the function $s \mapsto \ell\left(e^{s}\right)$ is periodic with period $\ln \left(\beta^{-2}\right)$. In fact, it can be proved that the same holds if $\mathbb{R}_{+}$is replaced by $\mathbb{C} \backslash(-\infty, 0]$.

- We would like to stress that the hierarchical model discussed here is not a mean-field model, and that the infinite-volume measure associated with the function $h_{I R}$ is an exact fixed point for the Wilson-Kadanoff transformation, without any approximations. The "only" flaw of this model is the lack of translation invariance and its consequences (no anomalous dimension). Apart from that, the model fully supports the general $R G$ picture, down to the numerical value of the critical index of the free 
energy ( $\nu=0.6495704 \ldots$ from non-rigorous numerical computation), which differs only by $2 \%$ from what is believed to be the correct value for translation-invariant models such as the Ising model in $d=3$ dimensions $(\nu \approx 0.638)$.

\section{Canonical Products and $\mathscr{N}$}

In this section, we will use Theorem 1.6 in order to show that the gaps for an $\mathscr{N}$-invariant canonical product of the form (1.9) decrease like $\nu_{k}-\nu_{k-1}=G\left(k^{-p}\right)$, if a finite number of these gaps satisfy a certain bound.

In addition to the convolution operators $H_{\lambda}$ defined in Eq. (1.12), consider now also the map $Q_{\beta}: f \mapsto f^{2}(\beta$.). Let $f \in \mathscr{E}$, and assume that the zeros $\omega_{k}(f)=\mu(f)+i \nu_{k}(f)$ of $f$ have been indexed by a set $I$ of consecutive integers (the intersection of $\mathbb{Z}$ with a connected subset of $\mathbb{R}$ ), such that the gaps defined by Eq. (1.13) are non-negative. Then we will index the zeros of $Q_{\beta}(f)$ by setting

$$
\omega_{2 k}\left(Q_{\beta}(f)\right)=\omega_{2 k+1}\left(Q_{\beta}(f)\right)=\beta^{-1} \omega_{k}(f), \quad \forall k \in I .
$$

With this convention, it is clear that $Q_{\beta}$ is order-preserving, in the same sense as the operators $H_{\lambda}$. Thus, since $\mathscr{N}^{\cdot}=H_{\lambda} \circ Q_{\beta}$ for $\lambda=\left(\beta^{-2}-1\right) / 4$, we obtain the following as a consequence of Lemma 1.4 and Theorem 1.6.

Corollary 2.1. Let $f$ and $g$ be functions in $\mathscr{E}$ with indexed zeros. Then $\mathscr{N}(f)$ and $\mathscr{N}(g)$ are also functions in $\mathscr{E}$, and if their zeros (which are all simple) are indexed as described above, then $\gamma(f) \prec \gamma(g)$ implies $\gamma(\mathscr{N}(f)) \prec \gamma(\mathscr{N}(g))$.

In order to take advantage of this property of $\mathscr{N}$, we need a class of functions $g$ such that the gaps for $\mathscr{N}(g)$ can be estimated. The following functions $h_{\ell}^{ \pm}$, and their translates, turn out to be ideal for this purpose.

Definition 2.2. For any fixed $\kappa>0$, define $h^{+}=\cosh \left(\kappa \pi\right.$.), $h^{-}=\sinh (\kappa \pi$.), and define $h_{0}^{ \pm}, h_{1}^{ \pm}, \ldots$ by the equation

$$
\begin{array}{ll}
h_{\ell}^{+}(z)=\prod_{k=0}^{\ell}\left[1+\frac{\kappa^{2} z^{2}}{(k+1 / 2)^{2}}\right], & z \in \mathbb{C}, \\
h_{\ell}^{-}(z)=\kappa \pi z \prod_{k=1}^{\ell+1}\left[1+\frac{\kappa^{2} z^{2}}{k^{2}}\right], & z \in \mathbb{C} .
\end{array}
$$

Notice that $h_{\ell}^{ \pm} \rightarrow h^{ \pm}$pointwise, as $\ell$ tends to infinity. The image under $\mathscr{N}$ of the limit can be computed explicitly:

$$
\left(\mathscr{N}\left(h^{ \pm}\right)\right)(z)=\frac{1}{2} e^{\left(1-\beta^{2}\right) \kappa^{2} \pi^{2}}\left[\cosh (2 \kappa \pi \beta z) \pm e^{-\left(1-\beta^{2}\right) \kappa^{2} \pi^{2}}\right] .
$$

The important observation here is that for large values of $\kappa$, the gaps between the zeros of $\mathscr{N}\left(h^{ \pm}\right)$are approximately a factor of $2 \beta=2^{1 / 6}$ smaller than the gaps between the zeros of $h^{ \pm}$. The following proposition will be used to show that the same is true for the "middle" gaps for $\mathscr{N}\left(h_{\ell}^{ \pm}\right)$, if $\ell$ is sufficiently large. Define

$$
a(\kappa)=\left(1-\beta^{2}\right) \exp \left(\frac{\beta^{2}}{4 \kappa^{2}\left(1-\beta^{2}\right)}\right)\left[\kappa^{-2}+2\left(1-\beta^{2}\right) \pi^{2}+\kappa^{-2} e^{-\left(1-\beta^{2}\right) \kappa^{2} \pi^{2}}\right] .
$$


Proposition 2.3. Let $\kappa>0$ and $\ell \in \mathbb{N}$. If $t$ is a real number that satisfies $|t| \leq \frac{1}{2 \kappa}$, then

$$
\left|\left(\mathscr{N}\left(h^{ \pm}\right)\right)(i t)-\left(\mathscr{N}\left(h_{\ell}^{ \pm}\right)\right)(i t)\right| \leq \frac{a(\kappa) \kappa^{4}}{\ell+1} \cdot \frac{1}{2} e^{\left(1-\beta^{2}\right) \kappa^{2} \pi^{2}} .
$$

Proof. Let $\sigma=$ " + ". We estimate first the difference between the function $\phi_{\ell}=$ $\left[h_{\ell}^{\sigma} / h^{\sigma}\right]^{2}$ and the constant function 1 . For real values of $s$, we obtain

$$
\begin{aligned}
\left|\phi_{\ell}^{\prime}(s)\right| & =\left|\frac{d}{d s} \prod_{k=\ell+1}^{\infty}\left[1+\frac{\kappa^{2} s^{2}}{(k+1 / 2)^{2}}\right]^{-2}\right| \\
& =\left|\sum_{k=\ell+1}^{\infty} \frac{-4 \kappa^{2} s}{(k+1 / 2)^{2}}\left[1+\frac{\kappa^{2} s^{2}}{(k+1 / 2)^{2}}\right]^{-1} \phi_{\ell}(s)\right| \\
& \leq 4 \kappa^{2}|s| \sum_{k=\ell+1}^{\infty} \frac{1}{(k+1 / 2)^{2}} \leq \frac{4 \kappa^{2}|s|}{\ell+1}
\end{aligned}
$$

and thus

$$
\left|\phi_{\ell}(s)-1\right|=\left|\int_{0}^{s} d t \phi_{\ell}^{\prime}(t)\right| \leq \frac{4 \kappa^{2}}{\ell+1} \int_{0}^{|s|} d t t=\frac{2 \kappa^{2} s^{2}}{\ell+1} .
$$

By using this last bound, the left-hand side of (2.5) can be estimated as follows:

$$
\begin{aligned}
\text { 1.h.s. } & =\left|\frac{1}{\sqrt{\left(1-\beta^{2}\right) \pi}} \int_{-\infty}^{\infty} d s e^{-\frac{1}{1-\beta^{2}}(s-i \beta t)^{2}}\left(h^{\sigma}(s)\right)^{2}\left[1-\phi_{\ell}(s)\right]\right| \\
& \leq \frac{1}{\sqrt{\left(1-\beta^{2}\right) \pi}} \int_{-\infty}^{\infty} d s\left|e^{-\frac{1}{1-\beta^{2}}(s-i \beta t)^{2}}\right|(\cosh (\kappa \pi s))^{2} \frac{2 \kappa^{2} s^{2}}{\ell+1} \\
& =\frac{1}{2} e^{\left(1-\beta^{2}\right) \kappa^{2} \pi^{2}}\left(1-\beta^{2}\right) \frac{\kappa^{2}}{\ell+1} e^{\frac{\beta^{2} t^{2}}{1-\beta^{2}}}\left[1+2\left(1-\beta^{2}\right) \kappa^{2} \pi^{2}+e^{-\left(1-\beta^{2}\right) \kappa^{2} \pi^{2}}\right] .
\end{aligned}
$$

After substituting the maximum value $\frac{1}{2 \kappa}$ for $|t|$, we get precisely the bound given in (2.5). The proof for the case $\sigma=$ "_" is similar.

The resulting bound on the middle gaps for $\mathscr{N}\left(h_{\ell}^{ \pm}\right)$will now be formulated more generally, for functions that have a sufficiently long sequence of gaps of size $\leq \kappa^{-1}$.

Definition 2.4. If $g$ is a function in $\mathscr{E}$ with indexed zeros and gaps $\gamma_{j}(g)$, we define for every $k \in \mathbb{Z}$ and $\ell \in \mathbb{N}$,

$$
\hat{\gamma}_{k}(g, \ell)=\max \left\{\gamma_{j}(g): k-\ell \leq j \leq k+\ell+1\right\} .
$$

Definition 2.5. Define $\mathscr{C b}$ to be the set of all triples $(\kappa, \theta, \ell)$ in $\mathbb{R}_{+} \times \mathbb{R}_{+} \times \mathbb{N}$ such that

$$
\frac{1}{2 \beta}+\theta<1, \quad e^{-\left(1-\beta^{2}\right) \kappa^{2} \pi^{2}}+\frac{a(\kappa) \kappa^{4}}{\ell+1}<\sin (\pi \beta \theta) .
$$


Proposition 2.6. Let $(\kappa, \theta, \ell) \in \mathscr{U b}$. Then for every $f \in \mathscr{E}$ with indexed zeros, and for every $k \in \mathbb{Z}$, the following holds:

$$
\hat{\gamma}_{k}(f, \ell) \leq \kappa^{-1} \quad \Longrightarrow \quad \hat{\gamma}_{2 k}(\mathscr{N}(f), 0) \leq\left(\frac{1}{2 \beta}+\theta\right) \kappa^{-1}
$$

Proof. Consider a fixed $k \in \mathbb{Z}$. Then, for any given $\ell \in \mathbb{N}$, we index the zeros of $h_{\ell}^{+}$ and $h_{\ell}^{-}$in increasing order of their imaginary parts, by successive integers ranging from $k-\ell-1$ up to $k+\ell$ and $k+\ell+1$, respectively. Define $t=\frac{1}{2 \kappa}\left(\frac{1}{2 \beta}+\theta\right)$.

We claim that under the given assumptions,

$$
\gamma_{2 k}\left(\mathscr{N}\left(h_{\ell}^{+}\right)\right) \leq 2 t, \quad \gamma_{2 k+1}\left(\mathscr{N}\left(h_{\ell}^{-}\right)\right) \leq 2 t
$$

Notice that the gaps considered in (2.12) are the ones between the two zeros of $h_{\ell}^{ \pm}$ that lie closest to the origin. By assumption, we have

$$
\cos (2 \kappa \pi \beta t)+e^{-\left(1-\beta^{2}\right) \kappa^{2} \pi^{2}} \leq-\frac{a(\kappa) \kappa^{4}}{\ell+1},
$$

and $|t| \leq \frac{1}{2 \kappa}$. Thus, it follows from Eq. (2.3) and Proposition 2.3 that

$$
\left(\mathscr{N}\left(h^{ \pm}\right)\right)(i t) \leq-\frac{a(\kappa) \kappa^{4}}{\ell+1} \cdot \frac{1}{2} e^{\left(1-\beta^{2}\right) \kappa^{2} \pi^{2}} \leq\left(\mathscr{N}\left(h^{ \pm}\right)\right)(i t)-\left(\mathscr{N}\left(h_{\ell}^{ \pm}\right)\right)(i t)
$$

which implies that $\left(\mathscr{N}\left(h_{\ell}^{ \pm}\right)\right)(i t) \leq 0$. Since the function $\left(\mathscr{N}\left(h_{\ell}^{ \pm}\right)\right)(i$.) is even and takes a positive value at the origin, we conclude that it has at least two zeros in the interval $[-t, t]$. This proves the bounds (2.12).

Assume now that $\hat{\gamma}_{k}(f, \ell) \leq \kappa^{-1}$. Then, since the (finite) gaps for the functions $h_{\ell}^{ \pm}$are all of size $\kappa^{-1}$, we have $\gamma(f) \prec \gamma\left(h_{\ell}^{ \pm}\right)$. But by Corollary 2.1 , this implies that $\gamma_{j}(\mathscr{N}(f)) \leq \gamma_{j}\left(\mathscr{N}\left(h_{\ell}^{ \pm}\right)\right)$for all $j$. Thus, the assertion follows from the bounds (2.12).

The statement (2.11) will now be used to inductively estimate the gaps for a fixed point $f$ of $\mathscr{N}$. Given the hypotheses of the lemma below, we can assume that the zeros $i \nu_{k}(f)$ of $f$ are indexed by $\mathbb{Z}$, in such a way that

$$
\nu_{k-1}(f) \leq \nu_{k}(f)=-\nu_{-k-1}(f), \quad \forall k \in \mathbb{Z} .
$$

Lemma 2.7. Let $f$ be an a non-constant even function in $\mathscr{E}$ that satisfies the fixed point equation for $\mathscr{N}$, and let $\left(\kappa_{0}, \theta, \ell_{0}\right)$ be an element of $\mathscr{W}$. If

$$
\gamma_{k}(f)<\kappa_{0}^{-1}, \quad n_{0}-\ell_{0} \leq k<2 n_{0},
$$

for some $n_{0}>\ell_{0}+1$, then for every positive $p<1 / 6$ there exists a constant $b$ such that

$$
\gamma_{k}(f)<b k^{-p}, \quad k=1,2 \ldots
$$

Proof. Assuming that the hypotheses of this lemma are satisfied, we shall first show that the conclusion holds for some (as opposed to all) positive $p<1 / 6$.

Depending on a choice of $p$, define two sequences $n \mapsto \kappa(n)$ and $n \mapsto \ell(n)$ as follows:

$$
\kappa(n)=\kappa_{0}\left(\frac{n}{n_{0}-\ell_{0}}\right)^{p}, \quad \ell(n)=\left[\ell_{0}\left(\frac{n}{n_{0}}\right)^{5 / 6}\right\rfloor,
$$


where $\lfloor r\rfloor$ denotes the integer part of a real number $r$. Below we will prove that

$$
(\kappa(n-\ell(n)), \theta, \ell(n)) \in \mathscr{C}
$$

for every $n \geq n_{0}$, provided that $p>0$ is sufficiently small. Our goal now is to show that for arbitrary $n \geq n_{0}$, if

$$
\gamma_{j}(f)<\kappa(j)^{-1}, \quad n-\ell(n) \leq j<2 n,
$$

then

$$
\gamma_{j}(f)<\kappa(j)^{-1}, \quad 2 n \leq j \leq 2 n+1 .
$$

This is sufficient to prove the assertion, since for $n=n_{0}$, the bound (2.20) follows from the assumption (2.16), if $p>0$ is sufficiently small.

Let now $n \geq n_{0}$ be fixed, and assume that (2.20) holds. Then $\hat{\gamma}_{n}(f, \ell(n))$ is less than or equal to $\kappa(n-\ell(n))^{-1}$, and we obtain the bound

$$
\hat{\gamma}_{2 n}(f, 0) \leq\left(\frac{1}{2 \beta}+\theta\right) \kappa(n-\ell(n))^{-1}
$$

from Proposition 2.6. Thus, in order to prove (2.21), it suffices to show that

$$
\frac{1}{2 \beta}+\theta<\frac{\kappa(n-\ell(n))}{\kappa(2 n+1)}, \quad \forall n \geq n_{0}
$$

To this end, choose an arbitrary positive real number $\varepsilon$. Then for every $n \geq n_{0}$, we have

$$
\begin{aligned}
\frac{\kappa(n-\ell(n))}{\kappa(2 n+1)} & =\left(\frac{n-\ell(n)}{2 n+1}\right)^{p}=2^{-p}\left(1-\frac{1}{2 n+1}\right)^{p}\left(1-\frac{\ell(n)}{n}\right)^{p} \\
& \geq 2^{-p}\left(1-\frac{1}{2 n_{0}+1}\right)^{p}\left(1-\frac{\ell_{0}}{n_{0}}\right)^{p}>2^{-p}(1-\varepsilon),
\end{aligned}
$$

if $p>0$ is sufficiently small. Thus, since the left-hand side of (2.23) is less than 1 by assumption, and since $\varepsilon$ was arbitrary, it follows that (2.23) holds for sufficiently small $p>0$. This shows that (2.20) implies (2.21).

In order to prove (2.19), choose again $\varepsilon>0$, and denote by $n_{1}$ the largest value of $n$ for which $\ell_{0}\left(n / n_{0}\right)^{1 / 2}<\ell_{0}+1$. Since the inequalities (2.10) in the definition of $\mathscr{U} 6$ are strict, the condition (2.19) holds for $n_{0} \leq n<n_{1}$, if $p$ is sufficiently small. And if $n \geq n_{1}$, we have the bound

$$
\begin{aligned}
\frac{\kappa(n-\ell(n))^{4}}{\ell(n)+1} & \leq \frac{\kappa_{0}^{4}}{\ell_{0}+1} \cdot \frac{\ell_{0}+1}{(\ell(n)+1)\left(n / n_{0}\right)^{-4 p}}\left(\frac{1}{1-\ell_{0} / n_{0}}\right)^{4 p} \\
& \leq \frac{\kappa_{0}^{4}}{\ell_{0}+1} \cdot \frac{\ell_{0}+1}{\ell_{0}\left(n / n_{0}\right)^{5 / 6}\left(n / n_{0}\right)^{-4 p}}\left(\frac{1}{1-\ell_{0} / n_{0}}\right)^{4 p} \\
& \leq \frac{\kappa_{0}^{4}}{\ell_{0}+1}\left(\frac{1}{1-\ell_{0} / n_{0}}\right)^{4 p} \leq \frac{\kappa_{0}^{4}}{\ell_{0}+1}(1+\varepsilon),
\end{aligned}
$$

for sufficiently small $p>0$. Thus, since the factor $a(\kappa)$ in (2.10) is a decreasing function of $\kappa$, we find that the condition (2.19) also holds for $n \geq n_{1}$, if $p$ is sufficiently small. 
After having proved (2.17) for some $p>0$, we will now choose new values for the parameters $\left(\kappa_{0}, \theta, \ell_{0}\right)$, and then proceed as above, but with "fixed $p$ and sufficiently large $n_{0}$ " instead of "fixed $n_{0}$ and sufficiently small $p$."

Given some positive $p<1 / 6$, let $\theta$ be a positive real number satisfying

$$
\frac{1}{2 \beta}+\theta<2^{-p}
$$

Then choose $\kappa_{0}$ and $\ell_{0}$ such that $\left(\kappa_{0}, \theta, \ell_{0}-1\right) \in \mathscr{Q} 6$, and define two sequences $n \mapsto \kappa(n)$ and $n \mapsto \ell(n)$ as in (2.18). Given any $\varepsilon>0$, a bound similar to (2.25) can be obtained for all $n \geq n_{0}$, if $n_{0}$ is chosen sufficiently large:

$$
\begin{aligned}
\frac{\kappa(n-\ell(n))^{4}}{\ell(n)+1} & \leq \frac{\kappa_{0}^{4}}{\ell_{0}} \cdot \frac{\ell_{0}}{\ell_{0}\left(n / n_{0}\right)^{5 / 6}\left(n / n_{0}\right)^{-4 p}}\left(\frac{1}{1-\ell_{0} / n_{0}}\right)^{4 p} \\
& \leq \frac{\kappa_{0}^{4}}{\ell_{0}}\left(\frac{1}{1-\ell_{0} / n_{0}}\right)^{4 p} \leq \frac{\kappa_{0}^{4}}{\ell_{0}}(1+\varepsilon) .
\end{aligned}
$$

Thus, if $n_{0}$ is sufficiently large, we have again (2.19) for all $n \geq n_{0}$. Under the same condition, the bound (2.20) holds in the case $n=n_{0}$, since $\gamma_{j}(f) \rightarrow 0$ as $j \rightarrow \infty$. And as shown before, (2.20) implies (2.21), provided e.g. that (2.23) is satisfied. But for sufficiently small $\varepsilon>0$, the inequality (2.23) follows from (2.26) and (2.24); and the latter holds for all $n \geq n_{0}$, if $n_{0}$ is chosen sufficiently large.

\section{Proof of Theorems $\mathbf{1 . 1}$ and $\mathbf{1 . 3}$}

The proofs given in this section use Lemma 2.7, together with the following two theorems. The first theorem is a reformulation of two results from [7, Sect. 4].

Theorem 3.1. Let $f \not \equiv 0$ be an even entire analytic fixed point for $\mathscr{N}$, whose restriction to $\mathbb{R}$ is real-valued, and whose Taylor coefficients (at the origin) are bounded in absolute value by those of the function $z \mapsto K \exp \left(\frac{1}{r} z^{2}\right)$, for some constants $K>0$ and $r>\left(4 \alpha^{2}-1\right) /\left(2 \alpha^{2}-1\right)$. Then $f$ is the Fourier transform of a positive measure on $\mathbb{R}$ whose moments are all finite, and there are constants $b_{1}$ and $b_{2}$ such that

$$
|f(z)| \leq b_{1} e^{b_{2}|z|^{6 / 5}}, \quad \forall z \in \mathbb{C} .
$$

Theorem 3.2. The transformation $\mathscr{N}$ has a fixed point $f^{*}$ with the following properties:

(a) $f^{*}$ is an even entire analytic function which takes real values when restricted to $\mathbb{R}$.

(b) The Taylor coefficients at zero of $f^{*}$ are bounded by the corresponding coefficients of the function $z \mapsto K \exp \left(\frac{1}{10} z^{2}\right)$, for some positive constant $K$.

(c) All zeros of $f^{*}$ of lie on the imaginary axis.

In addition, there are positive real numbers $y_{0}<y_{1}<\ldots<y_{79}$, such that

(d) $f^{*}\left(i y_{k}\right)=0$ for $k=0,1, \ldots, 79$.

(e) The function $f^{*}\left(i\right.$.) has exactly 5 zeros in the interval $\left[0, y_{4}\right]$.

(f) $y_{k}-y_{k-1}<\frac{4}{3}$, for $k=5,6, \ldots, 79$.

A proof of Theorem 3.2 is given in [8]. In addition, we verify in [8] that

$$
\left(\frac{3}{4}, \frac{1}{10}, 35\right) \in \mathscr{U},
$$

where $\mathscr{Q} 6$ is the set specified in Definition 2.5 . 
Proof of Theorem 1.3. First, we note that $\left(4 \alpha^{2}-1\right) /\left(2 \alpha^{2}-1\right)<4$. Let $f_{I R}=f^{*}$, where $f^{*}$ is the fixed point of $\mathscr{N}$ described in Theorem 3.2. Since the properties (a) and (b) of this fixed point (and (e), which implies that $f_{I R} \not \equiv 0$ ) verify the assumptions of Theorem 3.1, we obtain for $f=f_{I R}$ the bound (3.1) and the inequality $f(0)>0$. Thus, given that $f_{I R}$ is even and satisfies (c) as well, it follows from Hadamard's factorization theorem that $f_{I R}$ can be represented as a convergent canonical product of the form (1.9). The lower bound on $\nu_{k}$ in (1.10) is obtained from (3.1), by using the standard inequality which bounds the number of zeros of an entire function $f$ in the disk $|z| \leq r$ by the logarithm of the maximum modulus of $f$ on the disk $|z| \leq e r$, if $f(0)=1$.

Finally, if we set $\left(\kappa_{0}, \theta, \ell_{0}\right)=\left(\frac{3}{4}, \frac{1}{10}, 35\right)$ and $n_{0}=40$, then the last three statements in Theorem 3.2, together with (3.2), imply that the hypotheses of Lemma 2.7 are satisfied. But the conclusion of this lemma is precisely the upper bound on the gaps $\gamma_{k}=\nu_{k}-\nu_{k-1}$ in (1.10). Thus, Theorem 1.3 is proved.

In order to show that the function $f_{I R}$ decreases along the imaginary axis as claimed in (1.8), we need the following simple fact.

Proposition 3.3. If $h$ is a $\mathrm{C}^{n}$ function that has $n>0$ zeros in an interval $[0, x]$, then

$$
|h(x)| \leq \frac{x^{n}}{n !} \sup _{t \in[0, x]}\left|h^{(n)}(t)\right| \text {. }
$$

Proof. Let $t_{0}=x_{-1}=x$. For $k=0,1, \ldots, n-1$, if we define $x_{k}$ to be the largest zero of $h^{(k)}$ in $\left[0, x_{k-1}\right]$, then $h^{(k+1)}$ has at least $n-k-1$ zeros in $\left[0, x_{k}\right]$. Thus,

$\left|h\left(t_{0}\right)\right|=\left|\int_{x_{0}}^{t_{0}} d t_{1} \int_{x_{1}}^{t_{1}} d t_{2} \cdots \int_{x_{n-1}}^{t_{n-1}} d t_{n} h^{(n)}\left(t_{n}\right)\right| \leq \int_{0}^{t_{0}} d t_{1} \int_{0}^{t_{1}} d t_{2} \cdots \int_{0}^{t_{n-1}} d t_{n} \sup _{t \in[0, x]}\left|h^{(n)}(t)\right|$,

and the assertion follows.

Proof of Lemma 1.2. Let $f_{I R}$ be the fixed point of $\mathscr{N}^{\cdot}$ described in Theorem 1.3. Then the hypothesis (and hence the conclusion) of Theorem 3.1 is clearly satisfied for the function $f=f_{I R}$. Thus, the only thing that remains to be proved is the bound (1.8).

Given that $f_{I R}(i$.) is the Fourier transform of some finite measure $\mu$, we have $\left|f_{I R}(i t)\right| \leq\left|f_{I R}(0)\right|$ for every $t \in \mathbb{R}$. The same inequality holds for all even derivatives of $f_{I R}$, since the moments of $\mu$ are finite. Thus, by Cauchy's formula and (3.1), there are constants $d_{1}, d_{2}>0$ such that

$$
\left|\frac{1}{n !} f_{I R}^{(n)}(i t)\right| \leq\left|\frac{1}{n !} f_{I R}^{(n)}(0)\right| \leq d_{1}\left(d_{2} n\right)^{-5 n / 6}, \quad t \in \mathbb{R}, n \in 2 \mathbb{N} .
$$

Let us now choose an arbitrary $q \in\left(1, \frac{6}{5}\right)$. Let $q-\frac{1}{5}<r<1$, and define $p=(q-r) /(1+q-r)$. Since $p<1 / 6$, it follows from Theorem 1.3 that $\nu_{k} \leq d_{3} k^{1-p}+d_{4}$ for some positive constants $d_{3}, d_{4}$. By combining this inequality with the second bound in (1.10), we find $d_{5}>0$ such that if $t$ is sufficiently large and $\nu_{k}>t-t^{r}$, then

$$
\nu_{k}-\nu_{k-1} \leq a_{2}\left(\frac{\nu_{k}-d_{4}}{d_{3}}\right)^{-p /(1-p)} \leq \frac{t^{-q+r}}{2 d_{5}}
$$


Thus, the function $f_{I R}\left(i\right.$.) has $n \geq d_{5} t^{q}$ zeros in the interval $\left[t-t^{r}, t\right]$ for large $t$. Now we can apply Proposition 3.3 and the bound (3.4) in order to estimate the value of the function $h=f_{I R}\left(i\left(t-t^{r}+.\right)\right)$ at $x=t^{r}$. The result is that

$$
\left|f_{I R}(i t)\right| \leq d_{6} \exp \left(-d_{7} t^{q} \ln t\right), \quad t>0,
$$

for some positive constants $d_{6}$ and $d_{7}$.

Proof of Theorem 1.1. Let $f_{I R}$ be the function described in Lemma 1.2. By analytic continuation, we can write the fixed point property of $f_{I R}$ in the form

$$
\frac{1}{\sqrt{\left(1-\beta^{2}\right) \pi}} \int_{-\infty}^{\infty} d s e^{-\frac{1}{1-\beta^{2}} s^{2}} f_{I R}\left(i \beta^{-1}(t+s)\right)=f_{I R}(i t)^{2}, \quad t \in \mathbb{C} .
$$

To be more precise, consider the operator $S_{i}$ which maps an entire function $f$ to the function $f\left(i\right.$.). A group property of the heat flow $\lambda \mapsto H_{\lambda}$, which will be proved in the next section, implies that $S_{i}^{-1} H_{\lambda} S_{i} H_{\lambda} f=f$, for every entire function $f$ that satisfies (1.11). Thus, given the bound (1.7), it follows that (3.7) is equivalent to the equation $\mathscr{N}\left(f_{I R}\right)=f_{I R}$.

Let now $h_{I R}$ be the function defined by Eq. (1.5). From (1.8) it is clear that this function is entire analytic, and that it satisfies the bound (1.4). In order to prove (1.3), consider the Fourier transform $\psi$ of the function $t \mapsto f_{I R}(i t)^{2}$. Below we will show that $\psi$ satisfies the bound

$$
|\psi(t)| \leq d_{1} e^{-d_{2} t^{6}}, \quad t \in \mathbb{R},
$$

for some constants $d_{1}, d_{2}>0$. By Eq. (3.7), this leads to an analogous estimate for the Fourier transform of $f_{I R}$, from which the upper bound in (1.3) follows. Given now that $\mathscr{R}\left(h_{I R}\right)$ is well defined, it is easy to check that the function $h_{I R}$ satisfies the fixed point equation for $\mathscr{B}$. In addition, for every $t \in \mathbb{R}$ we have $h_{I R}(t) \geq 0$, since $f_{I R}$ is a function of positive type; and $h_{I R}(t)=0$ is excluded by the fact that $h_{I R}$ is a fixed point for $\mathscr{R}$.

We will now turn to the proof of the bound (3.8). From the fact that $f_{I R}$ is the Fourier transform of a positive measure, it follows that the function $\psi$ takes only non-negative values on $\mathbb{R}$. In addition, since $\psi$ and $-\psi^{\prime \prime}$ are themselves functions of positive type, we have the bounds

$$
|\psi(t)| \leq|\psi(0)|=b^{2}, \quad\left|\psi^{\prime \prime}(t)\right| \leq\left|\psi^{\prime \prime}(0)\right|=2 c^{2}
$$

for all $t \in \mathbb{R}$. The constants $b, c>0$ are defined by (3.9). Let now $t$ be an arbitrary real number larger than $2 b / c$, and define $a=\psi(t)^{1 / 2}$. Then the even moments of $\psi$ can be bounded from below as follows:

$$
\begin{aligned}
\int_{-\infty}^{\infty} d x x^{2 n} \psi(x) & \geq\left(t-\frac{a}{c}\right)^{2 n} \int_{0}^{a / c} d x[\psi(t+x)+\psi(t-x)] \\
& \geq\left(\frac{t}{2}\right)^{2 n} \int_{0}^{a / c} d x 2\left[a^{2}-c^{2} x^{2}\right]=\left(\frac{t}{2}\right)^{2 n} \frac{4 a^{3}}{3 c}
\end{aligned}
$$


On the other hand, we have a bound on the Taylor coefficients of the function $f_{I R}^{2}$, which follows from (1.7), and which implies that

$$
\int_{-\infty}^{\infty} d x x^{2 n} \psi(x)=\left|\left(f_{I R}^{2}\right)^{(2 n)}(0)\right| \leq\left(d_{3} n^{1 / 6}\right)^{2 n}, \quad n=1,2, \ldots,
$$

for some constant $d_{3}>0$. The two inequalities (3.10) and (3.11) can now be combined to yield an upper bound on $\psi(t)$ : For any $\delta>0$ with the property that $n=\delta t^{6}$ is a positive integer, we get

$$
\frac{4}{3 c} \psi(t)^{3 / 2}=\frac{4 a^{3}}{3 c} \leq\left(2 d_{3} \delta^{1 / 6}\right)^{2 \delta t^{6}}
$$

Thus, if $\delta$ is chosen appropriately, the bound (3.8) follows. This completes the proof of Theorem 1.1.

\section{The Heat Flow on $\mathscr{E}$}

Denote by $\mathscr{F}$ the vector space of all entire analytic functions $f$ that satisfy the bound (1.11). On $\mathscr{F}$ we define the following directed family of seminorms:

$$
\|f\|_{\rho}=\sup _{z \in \mathbb{C}} e^{-|z|^{2} / \rho}|f(z)|, \quad \rho>0 .
$$

Equipped with the natural topology defined by these seminorms, $\mathscr{F}$ is a Fréchet space. We note that a linear operator $L$ on $\mathscr{F}$ is continuous (bounded) if and only if for every $\rho>0$ there exist $c, r>0$ such that $\|L f\|_{\rho} \leq c\|f\|_{r}$ for every function $f \in \mathscr{F}$.

Examples of continuous linear operators on $\mathscr{F}$ are differentiation $D: f \mapsto f^{\prime}$, translations $T_{\lambda}: f \mapsto f(.-\lambda)$, and, as shown below, the operators $K_{n, m}$, defined by the equation

$$
\left(K_{n, m} f\right)(z)=\frac{1}{2 \pi i} \int_{\Gamma_{z}} \frac{z^{n} f(\zeta) d \zeta}{\zeta^{m}(\zeta-z)}, \quad n \leq m, z \in \mathbb{C} .
$$

Here, $\Gamma_{z}$ is a closed curve in $\mathbb{C}$ with winding number 1 with respect to the points 0 and $z$. The proof for the continuity of $D$ and $T_{\lambda}$ is similar to the proof of the following proposition.

Proposition 4.1. Let $n \leq m$ be nonnegative integers. Then the operator $K_{n, m}$ is continuous on $\mathscr{F}$, and for every $\rho>0$, it satisfies the bound

$$
\left\|K_{n, m} f\right\|_{\rho} \leq 2^{-m+1} e^{1 / \rho}\|f\|_{4 \rho}, \quad f \in \mathscr{F} .
$$

Proof. Let $f \in \mathscr{F}$ and $\rho>0$. Given any $z \in \mathbb{C}$, let $\Gamma_{z}$ be the positively oriented circle of radius $2\left(|z|^{2}+1\right)^{1 / 2}$ around the origin. Then for every $\zeta \in \Gamma_{z}$ we have $\left|z^{n} / \zeta^{m}\right| \leq 2^{-m},|\zeta-z| \geq \frac{1}{2}|\zeta|$, and

$$
|f(\zeta)| \leq\|f\|_{4 \rho} e^{\frac{1}{4 \rho}|\zeta|^{2}}=\|f\|_{4 \rho} e^{1 / \rho+|z|^{2} / \rho}
$$

Thus, we can bound the right-hand side of (4.2) by $\exp \left(|z|^{2} / \rho\right)$ times the righthand side of (4.3), as claimed. Since $f$ and $\rho$ were arbitrary, it follows that $K_{n, m}$ is continuous. 
We shall now consider the heat flow $\lambda \mapsto H_{\lambda}$ defined in (1.12), but extended to complex values of $\lambda$.

Definition 4.2. For every $f \in \mathscr{F}$ and for every $\lambda \in \mathbb{C}$, define a function $H_{\lambda} f$ by the equation

$$
\left(H_{\lambda} f\right)(z)=\hat{f}(\lambda, z) \equiv \frac{1}{\sqrt{4 \pi}} \int_{-\infty}^{\infty} d s e^{-s^{2} / 4} f(z-\sqrt{\lambda} s), \quad z \in \mathbb{C} .
$$

Notice that this definition is independent of the choice of the square root function, and that $\hat{f}$ is analytic in both of its arguments.

Proposition 4.3. Let $\lambda \in \mathbb{C}, \rho>0, f \in \mathscr{F}$, and define $r=\rho+4|\lambda|$. Then $H_{\lambda}$ is a continuous linear operator on $\mathscr{F}$, and it satisfies the following two bounds:

$$
\begin{gathered}
\left\|H_{\lambda} f\right\|_{\rho} \leq(r / \rho)^{1 / 2}\|f\|_{r} \\
\left\|H_{\lambda} f-f-\lambda f^{\prime \prime}\right\|_{\rho} \leq \frac{1}{2}|\lambda|^{2}(r / \rho)^{1 / 2}\left\|f^{\prime \prime \prime \prime}\right\|_{r} .
\end{gathered}
$$

Proof. The inequality (4.6) is obtained by replacing $f(z-\sqrt{\lambda} s)$ in Eq. (4.5) by the bound $\|f\|_{r} \exp \left(|z-\sqrt{\lambda} s|^{2} / r\right)$, and then computing the resulting Gaussian integral. The second inequality can be obtained from the first one by using the identity

$$
\left(H_{\lambda} f\right)(z)=f(z)+\lambda f^{\prime \prime}(z)+\lambda^{2} \int_{0}^{1} d t(1-t)\left(H_{t \lambda} f^{\prime \prime \prime \prime}\right)(z), \quad z \in \mathbb{C} .
$$

The following proposition shows, among other things, that the heat flow on the space $\mathscr{F}$ is invertible.

Proposition 4.4. $H_{\lambda_{1}} H_{\lambda_{2}} f=H_{\lambda_{1}+\lambda_{2}} f$, for every $f \in \mathscr{F}$ and $\lambda_{1}, \lambda_{2} \in \mathbb{C}$.

Proof. Given any positive integer $n$, consider the vector space $\mathscr{P}_{n}$ of all polynomials of degree $\leq n$, equipped with some norm. On this space, differentiation $D$ is a bounded linear operator. An explicit calculation shows that $\lambda \mapsto H_{\lambda}, \lambda \geq 0$, is a semigroup of linear operators on $\mathscr{F}$ which leave $\mathscr{P}_{n}$ invariant. Thus, it follows from (4.7) that $H_{\lambda} f=\exp \left(\lambda D^{2}\right) f$, for every $f \in \mathscr{P}_{n}$ and $\lambda \geq 0$. By analyticity, the same holds for every $\lambda \in \mathbb{C}$. This proves the assertion in the case where $f$ is a polynomial. But polynomials are dense in $\mathscr{F}$. This follows e.g. from the fact that $\mathrm{I}-K_{n, n}$ projects $\mathscr{F}$ onto $\mathscr{P}_{n-1}$, and that $K_{n, n} f$ converges to zero as $n \rightarrow \infty$, for every $f \in \mathscr{F}$. The latter is a consequence of Proposition 4.1. Thus, the assertion is proved.

Let us now consider the $\lambda$-dependence of the zeros for $H_{\lambda} f$. We start with properties that hold for every function $f \in \mathscr{F}$. The next proposition is an immediate consequence of the implicit function theorem, given the fact that the function $\hat{f}$, defined by Eq. (4.5), is entire analytic.

Proposition 4.5. Let $f \in \mathscr{F}$ and $\left(\lambda_{0}, z_{0}\right) \in \mathbb{C} \times \mathbb{C}$. If $H_{\lambda_{0}} f$ has a simple zero at $z_{0}$, then in some open polydisk $U \times V$ containing $\left(\lambda_{0}, z_{0}\right)$, the set of solutions $(\lambda, z)$ of the equation $\left(H_{\lambda} f\right)(z)=0$ is the graph of an analytic function from $U$ to $V$.

Proposition 4.6. Let $m \geq 1$, and denote by $h_{m}$ the $m^{\text {th }}$ Hermite polynomial. Then for every $\rho>0$ there exists a constant $K>0$ such that the following holds. If $f$ is a 
function in $\mathscr{F}$ that has a zero of order $m$ at the origin, and if $\varepsilon$ is a complex number of modulus $\leq 1$, then

$$
\left\|\hat{f}\left(-\frac{\varepsilon^{2}}{2}, \varepsilon .\right)-\frac{\varepsilon^{m}}{m !} f^{(m)}(0) h_{m}\right\|_{\rho} \leq K|\varepsilon|^{m+1}\|f\|_{8 \rho+16} .
$$

Proof. Let $|\varepsilon| \leq 1$, and for every $f \in \mathscr{F}$ define $S_{\varepsilon} f=f(\varepsilon$.). In order to prove the bound (4.9), we may assume that $f$ satisfies $z^{-m} f(z) \rightarrow 1$ as $z \rightarrow 0$. Then we can write

$$
\left(S_{\varepsilon} f\right)(z)=\varepsilon^{m} z^{m}+\varepsilon^{m+1} z^{m+1}\left(K_{0, m+1} f\right)(\varepsilon z),
$$

where $K_{0, m+1}$ is the operator defined by Eq. (4.2). For every $n \in \mathbb{N}$ and $z \in \mathbb{C}$, define $p_{n}(z)=z^{n}$. An explicit calculation shows that $H_{-1 / 2} p_{m}=h_{m}$. Thus, by using (4.10) and Proposition 4.1, we obtain the bound

$$
\begin{aligned}
\left\|S_{\varepsilon} H_{-\varepsilon^{2} / 2} f-\varepsilon^{m} h_{m}\right\|_{\rho} & =\left\|H_{-1 / 2} S_{\varepsilon} f-\varepsilon^{m} h_{m}\right\|_{\rho} \\
& =\left\|\varepsilon^{m+1} H_{-1 / 2}\left[p_{m+1} \cdot\left(S_{\varepsilon} K_{0, m+1} f\right)\right]\right\|_{\rho} \\
& \leq(1+2 / \rho)^{1 / 2}|\varepsilon|^{m+1}\left\|p_{m+1} \cdot\left(S_{\varepsilon} K_{0, m+1} f\right)\right\|_{\rho+2} \\
& \leq(1+2 / \rho)^{1 / 2}|\varepsilon|^{m+1}\left\|p_{m+1}\right\|_{2 \rho+4}\left\|S_{\varepsilon} K_{0, m+1} f\right\|_{2 \rho+4} \\
& \leq K|\varepsilon|^{m+1}\|f\|_{8 \rho+16}
\end{aligned}
$$

for some positive constant $K$.

Proposition 4.7. Let $\omega$ and $r$ be continuous functions on some compact set $\Lambda \subset \mathbb{C}$, with values in $\mathbb{C}$ and $[0, \infty)$, respectively. Furthermore, let $f \in \mathscr{F}$, and denote by $m(\lambda)$ the number of zeros of $H_{\lambda} f$ in the disk $|z-\omega(\lambda)| \leq r(\lambda)$. Here, and in what follows, zeros of order $m$ are counted as $m$ zeros. Then, given any sufficiently small $\varepsilon>0$, there exists an open neighborhood $U$ of $f$ in $\mathscr{F}$, such that for every $g \in U$ and for every $\lambda \in \Lambda$, the function $H_{\lambda}$ g has exactly $m(\lambda)$ zeros in the disk $|z-\omega(\lambda)|<r(\lambda)+\varepsilon$.

Proof. For every $\delta>0$ define $U(\delta)=\left\{g \in \mathscr{F}:\|f-g\|_{\rho}<\delta\right\}$, where $\rho$ is the maximum value of $1+4|\lambda|$ on $\Lambda$. The assertion follows by a standard argument, if we can show that for every sufficiently small $\varepsilon>0$ there exists a $\delta>0$, such that a bound of the form

$$
\left|\left(H_{\lambda} f\right)(z)-\left(H_{\lambda} g\right)(z)\right|<\frac{1}{2} c_{\varepsilon}<\left|\left(H_{\lambda} f\right)(z)\right|
$$

holds on the circle $C_{\varepsilon}(\lambda)$ of radius $r(\lambda)+\varepsilon$ centered at $\omega(\lambda)$, for every $g \in U(\delta)$ and for every $\lambda \in \Lambda$. Consider first the second inequality in (4.12). Since the functions $\omega$ and $r$ are continuous, it follows that the set $C_{\varepsilon}=\left\{(\lambda, z): \lambda \in \Lambda, z \in C_{\varepsilon}(\lambda)\right\}$ is compact for every $\varepsilon \geq 0$. Thus, the continuous function $|\hat{f}|$ has a minimum value $c_{\varepsilon}$ on $C_{\varepsilon}$, and $c_{\varepsilon}$ is positive for sufficiently small values of $\varepsilon>0$. In order to get the first inequality in (4.12), we can use the bound

$$
\left|\left(H_{\lambda} f\right)(z)-\left(H_{\lambda} g\right)(z)\right| \leq \delta \sqrt{\rho} e^{|z|^{2}}, \quad g \in U(\delta),
$$

which follows from Proposition 4.3. Since $|z|$ is uniformly bounded on the compact set $C_{\varepsilon}$, it suffices to take $\delta$ small enough.

We shall now work towards a proof of Theorem 1.6, in the case where the functions involved have only a finite number of zeros. Here, only real values of the heat flow parameter $\lambda$ will be considered, unless specified otherwise. 
Definition 4.8. Denote by $\mathscr{E}_{f}$ the set of all functions in $\mathscr{E}$ that have only a finite number of zeros, and denote by $\mathscr{E}_{p}$ the set of all polynomials whose zeros all lie on the imaginary axis. In order to simplify notation, define $f_{\lambda}=H_{\lambda} f$.

Let $f$ be a function in $\mathscr{E}_{f}$. Then by a standard argument, there exists a polynomial $g \in \mathscr{E}_{p}$ such that

$$
f(z)=e^{b z} g(z-\mu), \quad z \in \mathbb{C},
$$

for some constants $b \in \mathbb{C}$ and $\mu \in \mathbb{R}$. A short calculation shows that

$$
f_{\lambda}(z)=e^{b^{2} \lambda} e^{b z} g_{\lambda}(z-\mu+2 b \lambda), \quad z \in \mathbb{C},
$$

which implies that the zero sets for $f_{\lambda}$ and $g_{\lambda}$ are related to each other by a translation, for every $\lambda$. Thus, if we prove the assertion of Theorem 1.6 for polynomials in $\mathscr{E}_{p}$, then the same holds for every function in $\mathscr{E}_{f}$.

Let now $g$ be a nonzero polynomial of degree $d \geq 2$. Then the functions $g_{\lambda}$ are polynomials of the same degree $d$, and we may write them in the canonical form

$$
g_{\lambda}(z)=a(\lambda) \prod_{k \in I}\left[z-i \nu_{k}\left(g_{\lambda}\right)\right], \quad z \in \mathbb{C} .
$$

Here, the zeros $i \nu_{k}\left(g_{\lambda}\right)$ of $g_{\lambda}$ have been indexed by some set $I$ of $d$ consecutive integers, in such a way that the curves $\lambda \mapsto \nu_{k}\left(g_{\lambda}\right)$ are continuous (not necessarily real-valued, at this point). That this is possible follows e.g. from the implicit function theorem and Proposition 4.6. Assume now that the $k^{\text {th }}$ zero of $g_{\lambda}$ is simple, for some given value $\lambda_{0}$ of the parameter $\lambda$. Then, by differentiating the equation $0=g_{\lambda}\left(i \nu_{k}\left(g_{\lambda}\right)\right)$ with respect to $\lambda$, and using that $\frac{d}{d \lambda} g_{\lambda}=g_{\lambda}^{\prime \prime}$, we obtain the identity

$$
\frac{d}{d \lambda} \nu_{k}\left(g_{\lambda}\right)=i \frac{g_{\lambda}^{\prime \prime}\left(i \nu_{k}\left(g_{\lambda}\right)\right)}{g_{\lambda}^{\prime}\left(i \nu_{k}\left(g_{\lambda}\right)\right)}=\sum_{\jmath \in I \backslash\{k\}} \frac{1}{\nu_{k}\left(g_{\lambda}\right)-\nu_{\jmath}\left(g_{\lambda}\right)},
$$

for every $\lambda$ near $\lambda_{0}$. Notice that if all numbers $\nu_{k}\left(g_{\lambda}\right)$ are real and distinct from each other at $\lambda=0$, then the same remains true for all $\lambda>0$.

The following definition makes precise what we mean (e.g. in Theorem 1.6) by a function with indexed zeros.

Definition 4.9. A function in $\mathscr{E}$ with indexed zeros is a nonzero function $f \in \mathscr{E}$, together with a pair $(I, \varphi)$, consisting of a set I of integers and an onto map $\varphi$ from $I$ to $f^{-1}(0)$, with the following properties: For all $j$ and $k$ in $I$, if $j<k$ then $k-1 \in I$ and $\operatorname{Im} \varphi(k-1) \leq \operatorname{Im} \varphi(k)$; and for every zero $z$ of $f$, the cardinality of $\varphi^{-1}(z)$ is equal to the order of $z$.

Remark. To simplify notation, if a quantity $q$ depends on a function $f$ with indexed zeros $(I, \varphi)$, we will write $q(f)$ instead of $q((f,(I, \varphi)))$. In particular, the real and imaginary parts of $\varphi(k)$ will be denoted by $\mu(f)$ and $\nu_{k}(f)$, respectively. We note that if $f$ has only a finite number of zeros, then the function $\varphi$ is uniquely determined by the choice of $I$. In addition, $I$ can be reconstructed from the corresponding sequence of gaps, defined in Eq. (1.13).

Proposition 4.10. For every $\lambda \geq 0, H_{\lambda}$ maps $\mathscr{E}_{f}$ to $\mathscr{E}_{f}$, and the following holds. Let $g$ and $h$ be functions in $\mathscr{E}_{f}$ with indexed zeros, and assume that the zeros of $H_{\lambda} g$ and $H_{\lambda} h$ have been indexed by the same set of integers as the zeros of $g$ and $h$, respectively. Then $\gamma(g) \prec \gamma(h)$ implies $\gamma\left(H_{\lambda} g\right) \prec \gamma\left(H_{\lambda} h\right)$. 
Proof. As was mentioned earlier, it suffices to prove the assertion for polynomials $g, h \in \mathscr{E}_{p}$. We may also assume that $g$ is of degree $\geq 2$. Consider first the case where all zeros of $g$ and $h$ are simple. Then by Eq. (4.17), $g_{\lambda}$ and $h_{\lambda}$ lie in $\mathscr{E}_{p}$ for all $\lambda>0$. From the same equation it follows that

$$
\frac{d}{d \lambda} \gamma_{k}\left(g_{\lambda}\right)=\frac{2}{\gamma_{k}\left(g_{\lambda}\right)}+\gamma_{k}\left(g_{\lambda}\right) S_{k}\left(g_{\lambda}\right)
$$

where

$$
S_{k}\left(g_{\lambda}\right)=-\sum_{j \in I \backslash\{k-1, k\}}\left|\left[\nu_{j}\left(g_{\lambda}\right)-\nu_{k}\left(g_{\lambda}\right)\right]\left[\nu_{\jmath}\left(g_{\lambda}\right)-\nu_{k-1}\left(g_{\lambda}\right)\right]\right|^{-1} .
$$

Assume now that $\gamma(g) \prec \gamma(h)$. Consider the set $\Lambda$ of all $x \geq 0$ such that $\gamma\left(g_{\lambda}\right) \prec \gamma\left(h_{\lambda}\right)$ for all $\lambda \leq x$, and assume that this set contains a maximum value $\lambda_{0}$. Then there exists a non-empty set $K \subset \mathbb{Z}$ such that $\gamma_{k}\left(g_{\lambda_{0}}\right)=\gamma_{k}\left(h_{\lambda_{0}}\right)<\infty$ for all $k \in K$. At $\lambda=\lambda_{0}$ we have for all $k \in K$,

$$
\frac{d}{d \lambda}\left[\gamma_{k}\left(g_{\lambda}\right)-\gamma_{k}\left(h_{\lambda}\right)\right]=\gamma_{k}\left(g_{\lambda}\right)\left[S_{k}\left(g_{\lambda}\right)-S_{k}\left(h_{\lambda}\right)\right] \text {. }
$$

But $S_{k}(u) \leq S_{k}(v)$ whenever $\gamma(u) \prec \gamma(v)$, and the inequality is strict whenever $\gamma_{k}(u)=\gamma_{k}(v)$ and $\gamma(u) \neq \gamma(v)$. Thus, it follows that $\gamma\left(g_{\lambda}\right) \prec \gamma\left(h_{\lambda}\right)$ for all $\lambda$ in some interval $\left(\lambda_{0}, \lambda_{0}+\varepsilon\right)$. This contradicts the assumption that the set $\Lambda$ has a maximum.

In the case where $g$ has multiple zeros, the same argument can be used first to prove that $\gamma\left(g_{\lambda}^{(n)}\right) \prec \gamma\left(h_{\lambda}^{(n)}\right)$, where $g^{(n)}$ and $h^{(n)}$ are defined such that

$$
\nu_{\imath}\left(g^{(n)}\right)=\nu_{i}(g)+i / n, \quad \nu_{j}\left(h^{(n)}\right)=\nu_{j}(h)+j / n,
$$

for all zeros of $g$ and $h$, respectively. Taking $n \rightarrow \infty$, the assertion now follows from Proposition 4.7.

Consider now an arbitrary function $f$ in $\mathscr{E}$ with indexed zeros $(I, \varphi)$. By Hadamard's factorization theorem and a theorem of Lindelöf [9], $f$ can be represented as a convergent product

$$
f(z)=a e^{b z} \prod_{k \in I} G\left(\mu(f)+i \nu_{k}(f), z\right), \quad z \in \mathbb{C},
$$

where

$$
G(\omega, z)= \begin{cases}e^{z / \omega}[1-z / \omega], & \text { if } \omega \neq 0 \\ z, & \text { if } \omega=0\end{cases}
$$

For every $n \in \mathbb{N}$, denote by $I_{n}$ the intersection of $I$ with the set $\{-n,-n+1, \ldots, n\}$, and define the $n^{\text {th }}$ partial product $f_{n}$ for $f$ by the equation

$$
f_{n}(z)=a e^{b z} \prod_{k \in I_{n}} G\left(\mu(f)+i \nu_{k}(f), z\right), \quad z \in \mathbb{C} .
$$

In what follows, the zeros of $f_{n}$ are always assumed to be indexed by the set $I_{n}$.

Proposition 4.11. Let $f_{1}, f_{2}, \ldots$ be the partial products for a function $f \in \mathscr{E}$. Then $f_{n} \rightarrow f$ in the topology of $\mathscr{F}$.

Proof. The task is to show that $\left\|f-f_{n}\right\|_{\rho} \rightarrow 0$ for every $\rho>0$. We shall only consider the case $I=\{0,1,2, \ldots\}$ here; the other cases are either similar or trivial 
(if $I$ is finite). Let now $\rho>0$ be fixed, and let $N$ be some positive integer such that $\omega_{k}=\mu(f)+i \nu_{k}(f)$ is nonzero, for all $k \geq N$. By using that $\left|e^{z}(1-z)\right| \leq \exp \left(2|z|^{2}\right)$ for all $z \in \mathbb{C}$, we get for all $n \geq N$ the bound

$$
\left|f_{n}(z) / f_{N}(z)\right|=\prod_{k=N+1}^{n}\left|G\left(\omega_{k}, z\right)\right| \leq \exp \left(2|z|^{2} \sum_{k=N+1}^{n}\left|\omega_{k}\right|^{-2}\right) .
$$

From the convergence of the product in (4.22), it follows that the sum over all $k$ of $\omega_{k}^{-2}$ converges. But since all $\omega_{k}$ 's have the same real part, this implies that the sum $\sum_{k=0}^{\infty}\left|\omega_{k}\right|^{-2}$ converges as well. Consequently, if $N$ is chosen sufficiently large, we have $\left\|f_{n} / f_{N}\right\|_{4 \rho}=1$ whenever $n \geq N$, and thus

$$
\left\|f_{n}\right\|_{2 \rho}=\left\|f_{N} \cdot f_{n} / f_{N}\right\|_{2 \rho} \leq\left\|f_{N}\right\|_{4 \rho}\left\|f_{n} / f_{N}\right\|_{4 \rho}=\left\|f_{N}\right\|_{4 \rho} .
$$

Under the same condition on $N$ and $n$, we get now the following bound:

$$
\begin{aligned}
\left\|f-f_{n}\right\|_{\rho} & \leq \sum_{k=n+1}^{\infty}\left\|f_{k}-f_{k-1}\right\|_{\rho}=\sum_{k=n+1}^{\infty}\left\|\left(G\left(\omega_{k}, .\right)-1\right) f_{k-1}\right\|_{\rho} \\
& \leq \sum_{k=n+1}^{\infty}\left\|G\left(\omega_{k}, .\right)-1\right\|_{2 \rho}\left\|f_{k-1}\right\|_{2 \rho} \leq\left\|f_{N}\right\|_{4 \rho} \sum_{k=n+1}^{\infty}\left\|G\left(\omega_{k}, .\right)-1\right\|_{2 \rho} .
\end{aligned}
$$

The last sum in (4.27) converges and tends to zero as $n \rightarrow \infty$, since we can extract a factor $\omega_{k}^{-2}$ from the term

$$
G\left(\omega_{k}, z\right)-1=\omega_{k}^{-2}\left[z^{2}\left(K_{0,2} G(1, .)\right)\left(z / \omega_{k}\right)\right],
$$

and bound the $2 \rho$-norm of the remaining factor [ $\cdots]$ uniformly in $k$. The latter follows from Proposition 4.1, by using the fact that $\left|z / \omega_{k}\right|<|z|$ for sufficiently large $k$.

We are now ready to prove the first part of Lemma 1.4.

Proposition 4.12. Let $f$ be a function in $\mathscr{E}$, and let $\lambda>0$. Then the function $H_{\lambda} f$ lies in $\mathscr{E}$, has the same number (cardinality) of zeros as $f$, and all its zeros are simple.

Proof. Let $f$ be a function in $\mathscr{E}$ with indexed zeros, and let $f_{1}, f_{2}, \ldots$ be the partial products for $f$, defined in Eq. (4.24). By Proposition 4.10, the functions $H_{\lambda} f_{n}$ all lie in $\mathscr{E}_{f}$. And from Proposition 4.3 and Proposition 4.11 it follows that $H_{\lambda} f_{n} \rightarrow H_{\lambda} f$ in $\mathscr{F}$, and that the zeros of $H_{\lambda} f$ are limits of zeros of the functions $H_{\lambda} f_{n}$. Thus, the difference between any two zeros of $H_{\lambda} f$ lies in $i \mathbb{R}$, which proves that $H_{\lambda} f \in \mathscr{E}$.

The discussion after Definition 4.8 shows that the following holds for every nonnegative integer $d$, and for every $\lambda \in \mathbb{R}$ : If $f$ has $d$ zeros then $H_{\lambda} f$ has $d$ zeros. The converse is also true since $H_{\lambda}^{-1}=H_{-\lambda}$, by Proposition 4.4. Thus, the same holds for $d=\infty$ as well.

Assume now for contradiction that $H_{\lambda} f$ has a zero $z_{0}$ of multiplicity $m>1$, for $\lambda=\lambda_{0}>0$. Then by Proposition 4.6, the function $H_{\lambda} f$ has $m$ zeros close to $z_{0}$ for $\lambda$ near $\lambda_{0}$, and $2\lfloor\mathrm{~m} / 2\rfloor$ of these zeros approach $z_{0}$ on curves that are tangent to the line $z_{0}+\mathbb{R}$, as $\lambda \uparrow \lambda_{0}$. This contradicts the fact that $H_{\lambda} \in \mathscr{E}$ for all $\lambda>0$. Thus, the zeros of $H_{\lambda} f$ are simple for all $\lambda>0$.

Let $f$ be a function in $\mathscr{E}$ with indexed zeros $(I, \varphi)$. By using Proposition 4.6, together with the implicit function theorem, we can find, for every $k \in I$, a positive 
real number $\lambda_{k}$ and a continuous function $\lambda \mapsto \omega_{k}(f, \lambda)$ from $\left[0, \lambda_{k}\right)$ to $\mathbb{C}$, such that the following holds:

(a) $\omega_{k}(f, 0)=\varphi(k)$, for every $k \in I$.

(b) $\left(H_{\lambda} f\right)\left(\omega_{k}(f, \lambda)\right)=0$, for every $k \in I$ and for every $\lambda<\lambda_{k}$.

(c) $\operatorname{Im} \omega_{k-1}(f, \lambda) \leq \operatorname{Im} \omega_{k}(f, \lambda)$, whenever $\{k-1, k\} \subset I$ and $\lambda<\min \left(\lambda_{k-1}, \lambda_{k}\right)$. The functions $\omega_{k}(f,$.$) will be referred to as the local zero curves for f$.

Proposition 4.13. Let $f$ be a function in $\mathscr{E}$ with indexed zeros, let $\omega_{k}(f,$.$) be one of$ the local zero curves for $f$, and let $f_{1}, f_{2}, \ldots$ be the partial products for $f$. Then there exists $\varepsilon_{k}>0$ such that the zero curves $\omega_{k}\left(f_{n},.\right)$ for $f_{n}$ converge to $\omega_{k}(f,$.$) uniformly$ on $\left[0, \varepsilon_{k}\right]$.

Proof. By Proposition 4.6 there exists $\varepsilon_{k}>0$ and $n_{k} \in \mathbb{N}$, such that for every positive $\varepsilon \leq \varepsilon_{k}$ and for every $n \geq n_{k}$, the zero $\omega_{k}\left(f, \varepsilon^{2} / 2\right)$ lies within a distance $\varepsilon^{3 / 2}$ of $\omega_{k}\left(f_{n}, \varepsilon^{2} / 2\right)$, and all other zeros of $H_{\varepsilon^{2} / 2} f$ are at a distance $3 \varepsilon^{3 / 2}$ or more from $\omega_{k}\left(f, \varepsilon^{2} / 2\right)$. Given any positive $\varepsilon \leq \varepsilon_{k}$, we can now use Proposition 4.7 , with $\Lambda=$ $\left[\varepsilon^{2} / 2, \varepsilon_{k}\right]$ and $r \equiv 0$, in order to find $N \geq n_{k}$ such that $\left|\omega_{k}(f, \lambda)-\omega_{k}\left(f_{n}, \lambda\right)\right| \leq \varepsilon^{3 / 2}$ for every $\lambda \leq \varepsilon_{k}$ and for every $n \geq N$.

The proof of Lemma 1.4 is completed with the following proposition.

Proposition 4.14. Let $f$ be a nonzero function in $\mathscr{E}$, and assume that $\left(H_{\lambda_{0}} f\right)\left(z_{0}\right)=0$, for some $z_{0} \in \mathbb{C}$ and some $\lambda_{0}>0$. Then there exists a unique continuous function $\omega:[0, \infty) \rightarrow \mathbb{C}$ such that $\omega\left(\lambda_{0}\right)=z_{0}$, and such that $\left(H_{\lambda} f\right)(\omega(\lambda))=0$ for all $\lambda \geq 0$.

Proof. First, we note that the uniqueness follows from the fact that all zeros of $H_{\lambda} f$ are simple, if $\lambda$ is positive. Let now $V_{0}$ be the largest subinterval of $[0, \infty)$ containing $\lambda_{0}$, on which there exists a function $\omega$ with the desired properties. By Proposition 4.5 , the intersection of $V_{0}$ with $(0, \infty)$ is open.

Assume for contradiction that $0 \notin V_{0}$, and denote by $\lambda_{1}$ the largest real number that is smaller than any element of $V_{0}$. Since $\hat{f}$ is analytic, we must have $|\omega(\lambda)| \rightarrow \infty$ as $\lambda \downarrow \lambda_{1}$. Consider the function $g=H_{\lambda_{1}} f$. Given that $g$ has zeros (by Proposition 4.12), we can index them and choose a local zero curve $\omega_{k}(g,$.$) for g$. According to Proposition 4.13, there exists a positive $\delta<\lambda_{0}-\lambda_{1}$ such that for any given $\varepsilon>0$, the $k^{\text {th }}$ zero curve for the partial product $g_{n}$ satisfies the bound $\left|\omega_{k}(g, \lambda)-\omega_{k}\left(g_{n}, \lambda\right)\right|<\varepsilon$, whenever $n$ is larger than some number $n_{0}(\varepsilon)$. Define $\lambda_{2}=\lambda_{1}+\delta$. Then, by Proposition 4.7, there exists an $\varepsilon_{1}>0$ such that $H_{\lambda_{2}} f$ has no zero other than $\omega\left(\lambda_{2}\right)$ within a distance $\varepsilon_{1}$ of $\omega\left(\lambda_{2}\right)$, and such that for every positive $\varepsilon \leq \varepsilon_{1}$, the function $H_{\delta} g_{n}$ has a unique zero $\zeta_{n}$ in the disk $\left|z-\omega\left(\lambda_{2}\right)\right|<\varepsilon$, if $n$ is larger than some number $n_{1}(\varepsilon) \geq n_{0}(\varepsilon)$. In addition, we have $\zeta_{n}=\omega_{j_{n}}\left(g_{n}, \delta\right)$ for some index $j_{n}$, since all zero curves of $g_{n}$ are defined on $[0, \infty)$. In order to show that $j_{n+1}=j_{n}$ for large $n$, consider an open disk $D \in \mathbb{C}$, such that both $D$ and its closure contain the straight line segment $L$ connecting the two points $\omega\left(\lambda_{2}\right)$ and $\omega_{k}(g, \delta)$, but none of the zeros of $H_{\delta} g$ that lie outside $L$. By Proposition 4.7, there exists $n_{2} \in \mathbb{N}$ such that the function $H_{\delta} g_{n}$ has the same number of zeros in $D$ as the function $H_{\delta} g$, for all $n \geq n_{2}$. But since the (imaginary parts of the) zero curves of $g_{n}$ cannot cross, it follows that $j_{n}=j$ for some fixed index $j$, whenever $n$ is larger than $n_{1}\left(\varepsilon_{1}\right)$ and $n_{2}$. Now, we can use that by Proposition 4.9, $\gamma\left(H_{\lambda} g_{n+1}\right) \prec \gamma\left(H_{\lambda} g_{n}\right)$ for every $n$ and for every $\lambda \geq 0$. This shows that the sequence $n \mapsto\left|\omega_{j}\left(g_{n}, \lambda\right)-\omega_{k}\left(g_{n}, \lambda\right)\right|$ is non-increasing as $n \rightarrow \infty$, for every $\lambda$ in the interval $[0, \delta]$, i.e., that the functions $\omega_{j}\left(g_{n},.\right)$ remain bounded on this interval. On the other hand, if $\lambda=\delta$ then $\omega_{\jmath}\left(g_{n}, \lambda\right)$ converges to $\omega(g, \lambda)=\omega\left(f, \lambda_{1}+\lambda\right)$, as $n \rightarrow \infty$; and by Proposition 4.7 , the same 
holds for every positive $\lambda \leq \delta$. But this contradicts the assumption that $0 \notin V_{0}$, which implied that $|\omega(\lambda)| \rightarrow \infty$ as $\lambda \downarrow \lambda_{1}$.

A similar argument shows that the set $V_{0}$ cannot have a finite upper bound.

Proof of Theorem 1.6. Let $f$ and $g$ be functions in $\mathscr{E}$ with indexed zeros $(I, \varphi)$ and $(J, \psi)$, respectively. As we just proved, there exists a family (indexed by $I$ ) of continuous function $\lambda \mapsto \omega_{k}(f, \lambda)$ from the interval $[0, \infty)$ to $\mathbb{C}$, such that $\left(H_{\lambda} f\right)(z)=0$ if and only if $z=\omega_{k}\left(H_{\lambda} f\right)$ for some $k \in I$. An analogous statement holds for $g$, and for the partial products $f_{n}$ and $g_{n}$ for $f$ and $g$, respectively. In addition, we have $H_{\lambda} f_{n} \rightarrow H_{\lambda} f$ and $H_{\lambda} g_{n} \rightarrow H_{\lambda} g$ for every $\lambda \geq 0$, as a consequence of Proposition 4.11 and Proposition 4.3.

Let now $\lambda$ be a fixed positive real number, and assume that $\gamma(f) \prec \gamma(g)$. Then for every $n$ we have $\gamma\left(f_{n}\right) \prec \gamma\left(g_{n}\right)$, and $\gamma\left(H_{\lambda} f_{n}\right) \prec \gamma\left(H_{\lambda} g_{n}\right)$ follows from Proposition 4.10. But by Proposition 4.13 and Proposition 4,7, the zeros $\omega_{k}\left(H_{\lambda} f_{n}\right)$ and $\omega_{k}\left(H_{\lambda} g_{n}\right)$ converge to $\omega_{k}\left(H_{\lambda} f\right)$ and $\omega_{k}\left(H_{\lambda} g\right)$, respectively, as $n$ tends to infinity. Thus, we conclude that $\gamma\left(H_{\lambda} f\right) \prec \gamma\left(H_{\lambda} g\right)$.

\section{The Dyson-Baker Hierarchical Model}

Following parts of [3], we show in this section how the transformation $\mathscr{B}$ derives from the full RG transformation $\mathscr{T}$ for the Dyson-Baker hierarchical model in 3 dimensions, and how the latter relates to a general RG transformation for continuousspin lattice models. We do not consider any specific properties of the IR fixed point for $\mathscr{T}$, since the results of $[6$, Sect. 4$]$ are easy to adapt to the present situation, as the following discussion will show.

A statistical mechanics model of real-valued spins on a set $\Lambda \subset \mathbb{Z}^{d}$ may be represented by a parameterized family of measures $\mu$ on the space of all possible spin configurations $\phi \in \mathbb{R}^{\Lambda}$, such that the quantities of interest can be obtained from the partition function $\int d \mu$ by differentiating it with respect to the parameters of the family. The RG transformations considered below are maps $\mu \mapsto \tilde{\mu}$ with the property that $\int d \tilde{\mu} \equiv \int d \mu$, which can be used for an iterative computation of partition functions. Here, $\tilde{\mu}$ represents a model on a smaller set $\tilde{\Lambda}$, whose spins are averages of the original spins.

One of the standard RG transformations is obtained by choosing the following averaging operator $A$ on $\ell^{2}\left(\mathbb{Z}^{d}\right)$ :

$$
(A \phi)(y)=N^{-d / 2} \sum_{x:\lfloor x / N\rfloor=y} \phi(x),
$$

where $N$ is some integer larger than 1 , and where $\lfloor z\rfloor$ is the point in $\mathbb{Z}^{d}$ obtained from $z \in \mathbb{R}^{d}$ by taking the integer part of its coordinates. In order to simplify notation, assume now that $\Lambda=\mathbb{Z}^{d}$. This will lead to integrals that are not well defined, but the cure to this is well known; see e.g. [6]. In addition to $A$, we can also choose a Gaussian measure $\mu_{C}$, with mean zero and (some given) covariance $C$, which will become a trivial fixed point of the RG transformation. Whenever possible, a given spin model will now be represented by the function $F$, defined by the equation $d \mu(\phi)=d \mu_{C}(\phi) F(\phi)$. Let $\alpha>0$ be such that the operator $\Gamma$, determined by the equation

$$
C=\Gamma+\left(\alpha^{2} N\right)^{-d}\left(C^{-1} A C\right)^{*} C\left(C^{-1} A C\right)
$$


has no negative eigenvalues. Then the following defines a Wilson-Kadanoff type RG transformation $\mathscr{T}$ :

$$
(\mathscr{T}(F))(\phi)=\tilde{F}(\phi) \equiv \int d \mu_{\Gamma}(\psi) F\left(\left(\alpha^{2} N\right)^{-d / 2}\left(C^{-1} A C\right)^{*} \phi+\psi\right),
$$

where $\mu_{\Gamma}$ is the Gaussian measure on $\mathbb{R}^{\Lambda}$ with mean zero and covariance $\Gamma$. It is easy to check that $\int d \mu_{C} \tilde{F}$ is equal to $\int d \mu_{C} F$, as required. Thus, the partition function for $F$ may be computed by iterating the transformation $\mathscr{B}$. This is particularly useful in cases where $C$ is unbounded but $\Gamma$ is bounded.

In hierarchical models, the covariance $C$ is chosen in such a way that the integral $\int d \mu_{\Gamma}$ factorizes over blocks $B(y)=\{x \in \Lambda:\lfloor x / N\rfloor=y\}$. And if one wants to mimic the short-range Ising model, the matrix elements $C(x, y)$ should decay roughly like the two-point function of the Ising model; or like the kernel of the inverse Laplacian, if $\eta=0$. In order to define such a choice, consider the operators $U$ and $A_{0}$ on $\ell^{2}(\Lambda)$, given by

$$
\begin{aligned}
(U \phi)\left(x_{1}, x_{2}, \ldots, x_{d}\right) & =\phi\left(x_{2}, x_{3}, \ldots, x_{d}, x_{1}\right), \\
\left(A_{0} \phi\right)\left(y_{1}, y_{2}, \ldots, y_{d}\right) & =N^{-1 / 2} \sum_{x_{d}:\left\lfloor x_{d} / N\right\rfloor=y_{d}} \phi\left(y_{1}, y_{2}, \ldots, y_{d-1}, x_{d}\right),
\end{aligned}
$$

and for $n=1,2,3 \ldots$ let

$$
A_{n}=U^{-n+1} A_{0} U^{n-1}, \quad P_{n}=\left(A_{1}^{*} U^{-1}\right)^{n-1}\left(1-A_{1}^{*} A_{1}\right)\left(U A_{1}\right)^{n-1} .
$$

Notice that $A_{n} A_{n}^{*}=1$ for all $n$, which implies that the operators $A_{n}^{*} A_{n}$ are orthogonal projections. By using this fact, it is easy to check that the $P_{n}$ 's are also a family of orthogonal projections, and that $P_{m} P_{n}=\delta_{m n} P_{n}$ for all $m$ and $n$. Let now $C=C_{\infty}$, where

$$
C_{k}=(4 \sigma)^{-1} \sum_{n=1}^{k}\left(\alpha^{2} N\right)^{n-1} P_{n}
$$

for some fixed $\sigma>0$. Since $A_{d} A_{d-1} \cdots A_{1}=A$ and $A^{*} P_{n} A=P_{n+d}$, the covariance $C$ satisfies the equation

$$
C=\Gamma+\left(\alpha^{2} N\right)^{d} A^{*} C A,
$$

with $\Gamma=C_{d}$. A straightforward calculation shows that $C^{-1} A C=\left(\alpha^{2} N\right)^{d} A$, i.e., that $C=C_{\infty}$ and $\Gamma=C_{d}$ also satisfy (5.2). Using the decomposition of $A$ and $\Gamma$ given above, we can now write $\mathscr{T}=\mathscr{T}_{d} \circ \mathscr{T}_{d-1} \circ \ldots \circ \mathscr{T}_{1}$, where

$$
\left(\mathscr{T}_{n}(F)\right)(\phi)=\int d \mu_{\Gamma_{n}}(\psi) F\left(\alpha N^{1 / 2} A_{n}^{*} \phi+\psi\right)
$$

with $\Gamma_{n}=\frac{1}{4 \sigma}\left(1-A_{n}^{*} A_{n}\right)$. We note that if $\alpha=N^{1 / d-1 / 2}$, then the spectrum of $C$, as given by (5.6), has a scaling property analogous to that of the inverse Laplacian.

The property that distinguishes hierarchical models from more realistic models is the fact that Gibbs factors of the form

$$
F(\phi)=\prod_{x \in \Lambda} \alpha \sqrt{2 \pi / \sigma} h(\phi(x))
$$


are mapped to Gibbs factors of a similar form by $\mathscr{T}$. In the case considered here, the integral in (5.8) factorizes into a product of $(N-1)$-dimensional integrals associated with blocks of size $N$. In particular, it is easy to check that for $N=2$,

$$
\left(\mathscr{T}_{n} F\right)(\phi)=\prod_{x \in \Lambda} \alpha \sqrt{2 \pi / \sigma}(\mathscr{B} h)(\phi(x)),
$$

where $\mathscr{R}$ is the transformation given in (1.1), with $K=2 \alpha$.

\section{References}

1. Dyson, F.J.: Existence of a Phase Transition in a One-Dimensional Ising Ferromagnet. Commun. Math. Phys. 12, 91 (1969)

2. Wilson, K.: Renormalization Group and Critical Phenomena. II. Phase Space Cell Analysis and Critical Behavior. Phys. Rev. B4, 3184 (1971)

3. Baker, G.A.: Ising Model with a Scaling Interaction. Phys. Rev. B5, 2622 (1972)

4. For a more extensive list of references see [6]

5. Gallavotti, G.: Some Aspects of the Renormalization Problems in Statistical Mechanics. Memorie dell' Accademia dei Lincei 15, 23 (1978)

6. Koch, H., Wittwer, P.: A Non-Gaussian Renormalization Group Fixed Point for Hierarchical Scalar Lattice Field Theories. Commun. Math. Phys. 106, 495 (1986)

7. Koch, H., Wittwer, P.: On the Renormalization Group Transformation for Scalar Hierarchical Models. Commun. Math. Phys. 138, 537 (1991)

8. Koch, H., Wittwer, P.: Bounds on the Zeros of a Renormalization Group Fixed Point. In Preparation

9. See e.g. Theorem (2.10.3) in:

Boas, R.P.: Entire Functions. New York: Academic Press 1954

Communicated by J.-P. Eckmann.

This article was processed by the author

using the Springer-Verlag TEX CoMaPhy macro package 1991. 
